\title{
Radiological imaging and non-surgical local treatments for cholangiocarcinoma
}

\author{
Angelo Della Corte ${ }^{1,2}$, Ettore Di Gaeta ${ }^{1}$, Stephanie Steidler ${ }^{1}$, Francesco De Cobelli ${ }^{1,2,3}$ \\ 'Department of Radiology, IRCCS San Raffaele Scientific Institute, 20132 Milan, Italy. \\ ${ }^{2}$ Vita-Salute University, 20132 Milan, Italy. \\ ${ }^{3}$ Experimental Imaging Center, IRCCS San Raffaele Scientific Institute, 20132, Milan, Italy.
}

Correspondence to: Francesco De Cobelli, Department of Radiology, IRCCS San Raffaele Scientific Institute, Via Olgettina 60, Milan 20132, Italy. E-mail: decobelli.francesco@hsr.it

How to cite this article: Della Corte A, Di Gaeta E, Steidler S, De Cobelli F. Radiological imaging and non-surgical local treatments for cholangiocarcinoma. Hepatoma Res 2022;8:5. https://dx.doi.org/10.20517/2394-5079.2021.103

Received: 2 Aug 2021 First Decision: 16 Nov 2021 Revised: 30 Nov 2021 Accepted: 7 Dec 2021 Published: 13 Jan 2022

Academic Editors: Guang-Wen Cao, Gen-Sheng Feng Copy Editor: Xi-Jun Chen Production Editor: Xi-Jun Chen

\begin{abstract}
Cholangiocarcinoma (CC) is a malignancy with a very heterogeneous spectrum of morphopathological and prognostic characteristics. Diagnostic imaging is fundamental for early detection, preoperative staging, and resectability assessment, as well as early recognition of prognostic factors. Radical surgical treatment is limited by disease stage and technical feasibility. Interventional radiology has acquired a critical function in addressing disease control and survival improvement through loco-regional therapies, specifically in the setting of intrahepatic CC. In this review, we will describe the current state of art of diagnostic imaging, focusing on intrahepatic CC and proximal extrahepatic CC, and delineate the available loco-regional therapies strategies for unresectable intrahepatic CC.
\end{abstract}

Keywords: Cholangiocarcinoma, diagnostic imaging, loco-regional therapies, ablation, embolization

\section{INTRODUCTION}

Cholangiocarcinoma (CC) represents the most frequent malignant tumor of the biliary tract, accounting for $10 \%$ to $20 \%$ of all primary liver tumors ${ }^{[1]}$. These malignancies can be classified according to their morphological characteristics, anatomical site of origin, or based on histological classification. Even though histologically the vast majority are adenocarcinomas, with a high proportion of fibrous stroma, CCs represent a very diverse disease spectrum. 
Based on their anatomical localization, CCs are subdivided into intrahepatic cholangiocarcinoma (ICC) (i.e., originating from intrahepatic bile ducts) and extrahepatic cholangiocarcinoma (ECC). ECCs are further subdivided into perihilar- (peCC) and distal-extrahepatic cholangiocarcinoma (deCC) depending on its proximal or distal origin with respect to the confluence of the cystic duct into the main bile duct.

Morphologically, CCs are subdivided into mass-forming, periductal-infiltrating, or intraductal-growth, with intrinsic imaging findings and prognostic outcome. Intraductal-growth CCs tend to have a better prognosis, while mass-forming and periductal infiltrating types appear to have a poor outcome.

The global incidence of CC varies from 0.3 to 6 per 100,000 inhabitants per year ${ }^{[2]}$, and similarly, the mortality ranges from 1 to 6 inhabitants per 100,000 per year ${ }^{[3]}$. Both CC incidence and mortality have been increasing in the past few decades, representing a relevant health burden.

Risk factors for development of CC are present in approximately 50\% of cases. These risk factors vary according to geographical distribution as well as anatomical location. Diseases affecting the peripheral bile ducts, such as choledocholithiasis and primary sclerosing cholangitis, are more strongly associated to ECC; whereas, intrahepatic conditions, such as cirrhosis (i.e., viral or NASH-related) and intrahepatic lithiasis, are more typically linked to $\mathrm{ICC}^{[4]}$. Accordingly, patient age at diagnosis shows extreme variability, ranging from 2 nd decade of life in the case of Cairoli disease ${ }^{[5]}$ to the 6 th decade of life in NASH-related forms ${ }^{[6]}$. Comorbidities typical of CC patients, including but not limited to reduced liver function and cholestasis, may influence prognosis ${ }^{[7]}$, as well as treatment selection whenever they contribute to patient unfitness for surgery.

CC imaging requires in-depth knowledge of disease specific characteristics, as cases may present with atypical imaging features ${ }^{[8]}$ or "conventionally" described characteristics may mimic other malignancies or benign lesions ${ }^{[9,10]}$. Imaging evaluation of specific sub-group characteristics is therefore of paramount importance for disease diagnosis and therapeutic approach assessment. To date, computed tomography (CT) and magnetic resonance imaging (MRI), aided by magnetic resonance cholangiopancreatography (MRCP) sequences, remain the most commonly used imaging modalities. Positron emission tomography $(\mathrm{PET})^{[1,12]}$ may be useful for detection of metastases and may be of aid in further personalizing treatment options. In addition to diagnosis and resectability assessment, imaging may aid in providing information about prognosis of CCs, by combining information deriving from morphology, enhancement pattern, and texture analysis along with clinical and histopathological data.

Regarding treatment, surgical resection with negative margins is recognized as the only curative treatment for both ICC and $\mathrm{ECC}^{[13]}$. Several limitations, including disease stage, inadequate future liver remnant, presence of metastases, and technical contraindications, lower resectability rate to $20 \%-30 \%^{[14,15]}$. In patients with unresectable ICC, loco-regional treatment options are valid alternatives to systemic chemotherapy and have been shown to increase overall survival ${ }^{[16,17]}$. The most common techniques are ablative and intraarterial therapies. Recent evidence also supports these approaches and suggests a role for loco-regional treatments in the adjuvant and neo-adjuvant setting. Combined approaches can also be used when imaging and histopathology identify the need to confirm negative margins in patients with non-upfront surgical resection ${ }^{[18]}$. For ECC, the role of the interventional radiologist is mostly limited to the palliative and ancillary setting (i.e., biliary drainage/stent positioning and hypertrophy-inducing techniques to allow surgical resection), without direct effects on disease burden ${ }^{[19]}$. 
In this short review, we will describe the current state of art diagnostic methods and the prognostic value of imaging in ICC and peCC, as well as the loco-regional therapeutic strategies for unresectable ICC.

\section{DIAGNOSTIC IMAGING}

Imaging choice is essential for correct tumor assessment and for the best patient management prior to, during, and after therapy. A multimodality approach, which includes CT, MRI with MRCP and PET, is usually necessary to reach a final diagnosis and correctly identify and select patients who may undergo surgery [Figures 1-3]. Imaging has a high negative predictive value with respect to resectability ${ }^{[2,221]}$. Additional information may be collected using endoscopic retrograde cholangiopancreatography for completion of histological diagnosis if not already available.

\section{Multidetector computerd tomography}

Multiphasic contrast-enhanced CT scan has a well-documented role in diagnosis, tumor staging, and preoperative assessment of both peCC and $\mathrm{ICC}^{[9,22,23]}$. It also offers clinical information regarding local staging (e.g., relationship with hepatic ducts and vessels) and assessment of metastatic disease. The scan(s) should be obtained in the four phases: pre-contrast, arterial, portal, and delayed (3-5 min) post-contrast phases for evaluating enhancement patterns of the mass. The pre-contrast phase is useful in identifying predisposing peCC conditions such as intrahepatic stones, while post-contrast imaging ${ }^{[2]}$ is useful for differential diagnosis of ICC given its abundance in fibrous stroma.

CT is generally the most commonly used imaging for upfront staging, and it is the modality used to describe vascular and intrahepatic involvement such as relationship with hepatic ducts and vessels. Fusion imaging techniques and three-dimensional CT angiography are useful in planning surgery, reducing time of intervention ${ }^{[25]}$.

\section{MRI - MRCP}

Currently, MRI is the most common imaging method used to diagnose perihilar CC. Two-dimensional and three-dimensional (3D) MR cholangiography is considered to be the best non-invasive modality to evaluate the biliary system and correctly assess intraductal lesions. Heavily T2-weighted sequences, such as halfFourier acquisition single-shot turbo spin echo or single-shot fast spin echo, can be used to distinguish the hyperintense signal in the bile ducts from the remaining suppressed signal when performing MRCP. MRI protocol should include axial and coronal T2-weighted sequences, dynamic study using T1-fat suppressed sequences such as DIXON, and diffusion imaging. MRI is more sensitive than CT for the detection of intrahepatic metastasis and extremely useful in pre-operative assessment ${ }^{[2,2,2]}$. Gadoxetic acid-enhanced MRI, in particular, is particularly sensitive in the hepatobiliary phase ${ }^{[28,29]}$, due to hepatocyte uptake leading to clear parenchymal enhancement. In addition, diffusion-weighted (DWI) sequences, performed using 0$100 \mathrm{~s} / \mathrm{mm}^{2}$ and $800-1000 \mathrm{~s} / \mathrm{mm}^{2}$ for low- and high-b values, increases sensitivity of detection ${ }^{[30]}$.

Preoperative MRI is commonly performed in patients with CC in order to assess the extent, resectability, and vascular involvement of the tumor. It is also used to map hepatic vessels and identify vascular anatomic variants since an accurate preoperative assessment of liver vasculature has been shown to significantly affect the surgical outcome in patients with $\mathrm{CC}^{[31]}$.

Park et al. ${ }^{[32]}$ nearly two decades ago compared the diagnostic performance of MRCP with that of combined MDCT-angiography and found comparable results between the two techniques. MRCP sequences are usually acquired using both (radial) thick-slab- and thin 3D T2 sequences; the former provides a comprehensive overview of the biliary system with a good suppression of the surrounding tissue. Thin T2 

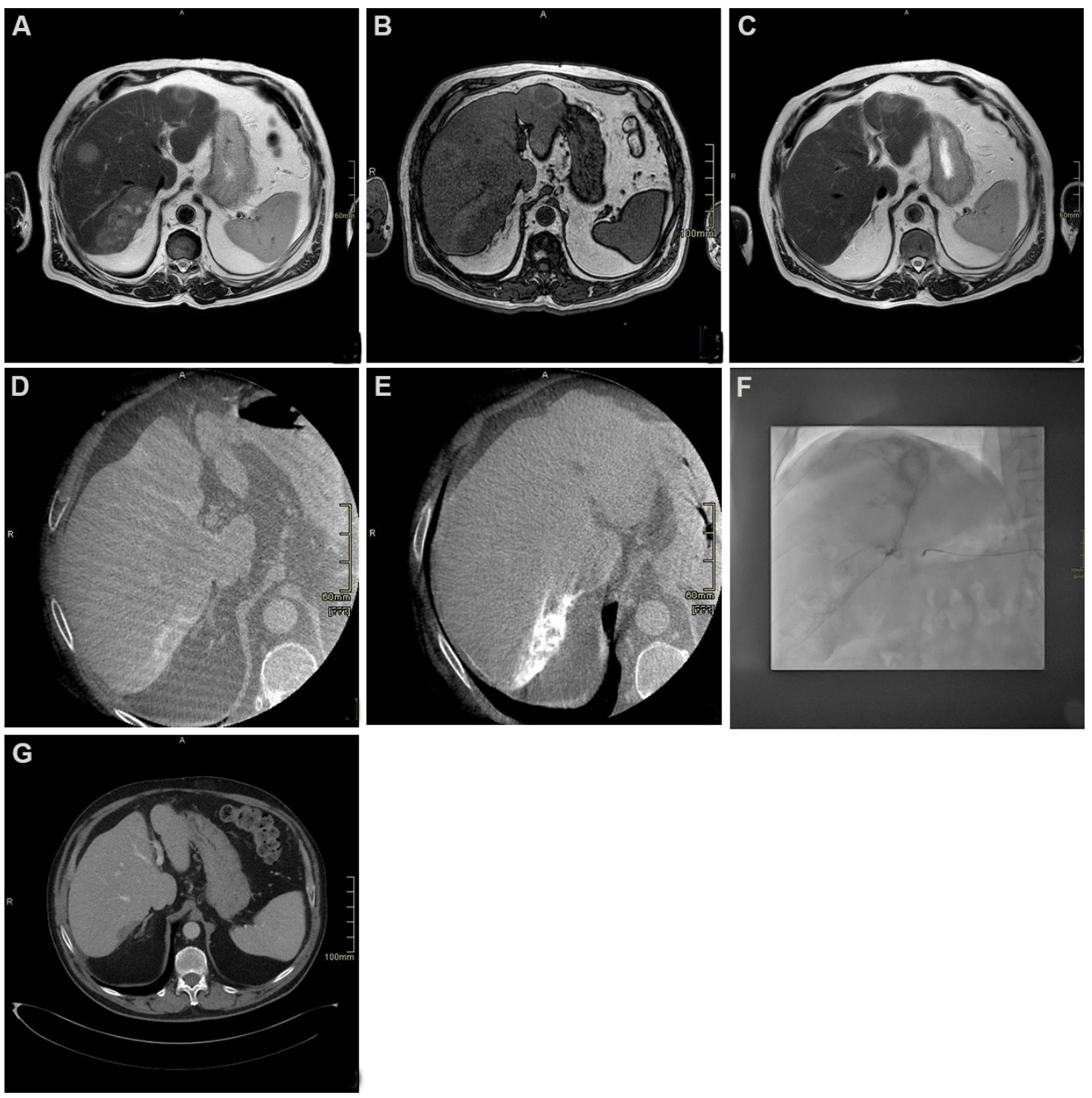

Figure 1. Seventy-year-old male patient with diagnosis of intrahepatic cholangiocarcinoma (ICC) undergoing chemotherapy and drugeluting beads transarterial chemoembolization. (A) T2 weighted axial scan demonstrating multifocal hyperintense ICC; (B) T1 weighted axial scan demonstrating multifocal hypointense ICC; (C) T2 weighted axial scan after chemotherapy showing significant reduction; (D) cone beam computed tomography (CT) arterial phase demonstrating target lesion for transarterial chemoembolization showing peripheral vascular "rim" enhancement; (E) cone beam CT after treatment showing uptake of embolizing beads; (F) angiogram during transarterial chemoembolization treatment, showing lesion uptake; (G) delayed post-contrast CT scan phase 1 month after treatment demonstrating complete devascularization of the lesion.

sequences, driven by thick slab sequences, provide detailed spatial resolution of complex anatomical structures and detection of small abnormalities such as small masses or stenosis ${ }^{[33]}$. In addition to MRCP, which is useful for depicting intraductal CC tumor growth, DWI-MRI, dynamic contrast-enhanced MRI, and late-phase sequences with hepatocyte-specific contrast agents can describe extra-ductal tumor growth as well as identify tumor masses located within dilated bile ducts ${ }^{[3,34]}$.

Imaging plays a central role in the preoperative staging and follow-up of CC, as well as in the surveillance of patients with an increased risk of CC. The lifetime incidence of CC in patients with primary sclerosing cholangitis ranges from $5 \%$ to $10 \%{ }^{[33]}$. According to recent recommendations, MRI including MRCP is 

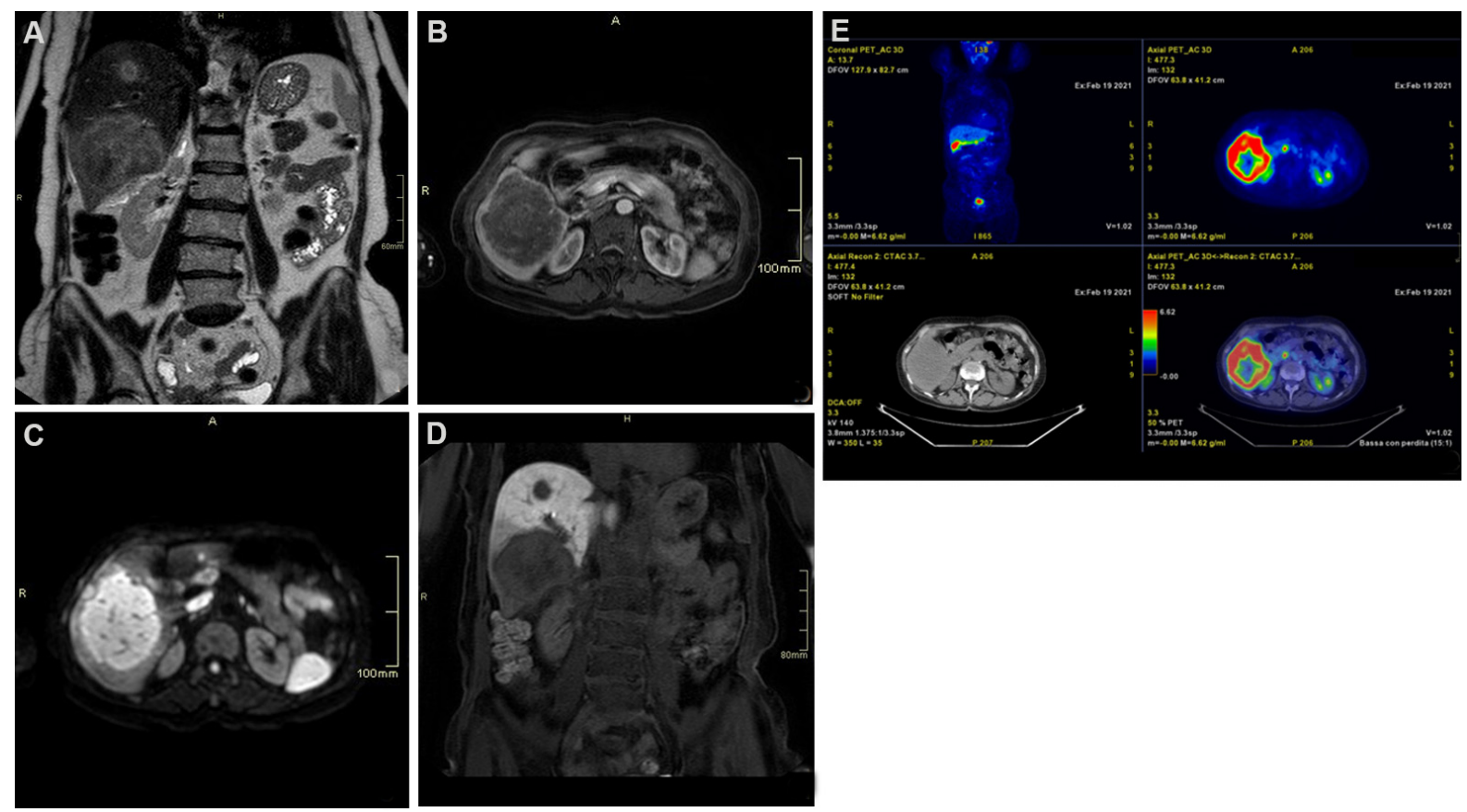

Figure 2. Seventy-one-year-old female patient with hepatitis C cirrhosis. (A) T2 weighted coronal scan demonstrating multifocal hyperintense intrahepatic cholangiocarcinoma; (B) T1 fat suppressed axial scan arterial phase showing peripheral rim enhancement; (C) diffusion-weighted scan demonstrating hyperintense signal; (D) T1 fat suppressed coronal scan showing hypointense signal on hepatobiliary phase: note the hypointense rim surrounding a relatively hyperintense cloud-like area; (E) 18F-FDG PET Scan demonstrating lesions uptake.
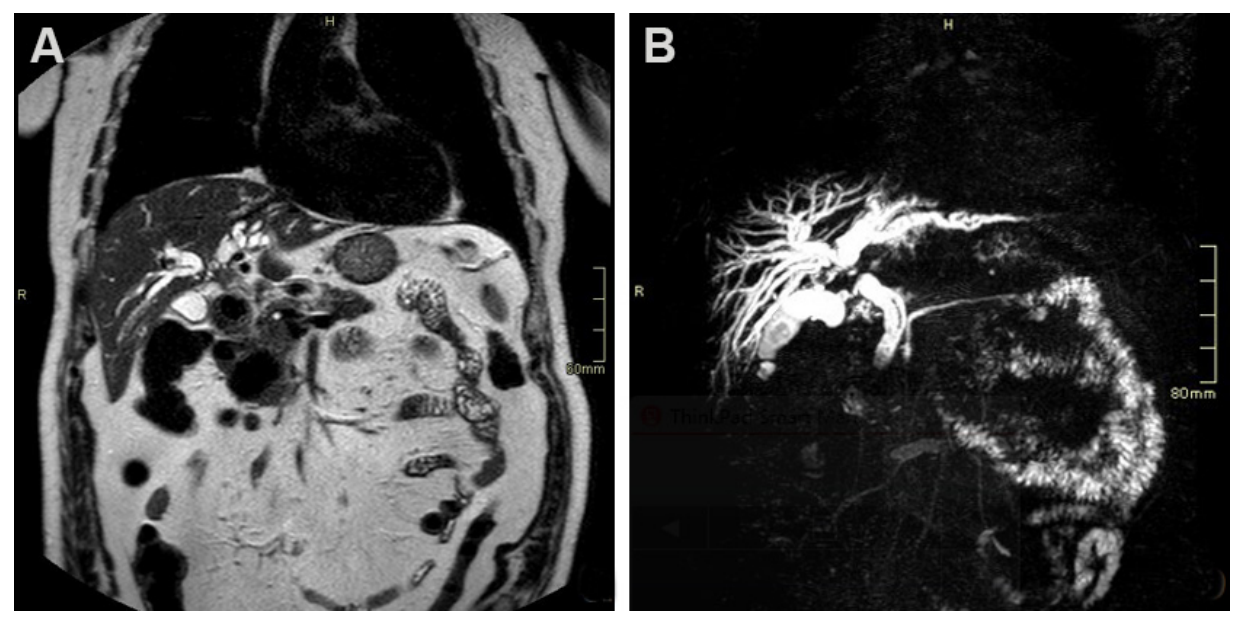

Figure 3. Seventy-nine-year-old male patient presenting with cholestasis. Coronal single shot fast spin echo T2 images (A) and magnetic resonance cholangiopancreatography $(B)$ show an infiltrating mass in the common bile duct (CBD), proximal to cystic duct insertion, leading to imaging diagnosis of perihilar cholangiocarcinoma. The patient was deemed resectable and successfully underwent right hepatectomy with $\mathrm{CBD}$ resection.

appropriate for the surveillance of these patients ${ }^{[35]}$.

\section{PET-CT/PET-MRI}

Combined modalities, such as PET/CT and PET/MRI, give both functional and morphological information on involvement and extension of disease. These modalities may be useful in pre-operative assessment as 
they allow detection of both distant and nodal metastasis using 18F-FDG. Potential caveats may be false positives in cases with biliary inflammation and false negatives in mucinous $\mathrm{CC}^{[36]}$.

peCC

The most common peCC is the periductal infiltrating type characterized by elongated strictures with irregularly visible walls on imaging and delayed enhanced duct dilation. Imaging assessment of tumor infiltration in the deeper mucosal layers of the bile ducts is challenging ${ }^{[37]}$. In addition, imaging characteristics of peCC are similar to those of other hepatic diseases, benign and malignant, and differential diagnosis becomes cardinal for correct patient management. In these cases, evaluation using contrast enchanced-CT or MRI-MRCP is recommended. DWI seems to be able to differentiate malignant strictures in extrahepatic disease ${ }^{[38]}$, with contrasting views of T1 sequences in peCC. 18F-FDG PET/CT has shown to be able to differentiate between benign and malignant strictures ${ }^{[3,40]}$ in larger lesions ${ }^{[1,42]}$.

Surgical assessment on imaging, even more in the light of new advances in surgical techniques and perioperative management which allow a larger cohort of patients to pursue this curative indication, is also challenging. Extent of involvement of the bile ducts (proximal margin involvement and longitudinal extent) and patient intrinsic patient characteristics are major differentials. Imaging using different CT modalities (i.e., CT alone, CT with direct cholangiography, or CT with reconstruction) have been able to confirm the documented ability of MRCP-dynamic MRI to identify secondary confluence involvement with comparable results ${ }^{[37]}$. The description of local extent of tumor invasion, anatomy of the biliary tree and association of tumor extent within the liver is mandatory for optimal resectability assessment.

\section{ICC}

Mass-forming ICCs are the most common morphological subtype of ICCs. On imaging they appear as large non-capsulated masses with associated peripheral dilation of bile ducts and may appear hypervascular similarly to HCC. Further characteristic imaging features include capsule retraction. Depending on their stromal component, generally characterized by fibrosis following desmoplastic reaction in the central portion, they may exhibit a typical late central enhancement with peripheral hypoenhancement. In several cases, additional nodules in proximity of the lobulated or irregular mass forming nodules and hepatic metastases are also visible. Since pattern of enhancement can vary, differential diagnosis with HCC is often challenging even when gadoxetic acid is used as contrast agent ${ }^{[43-45]}$. However, MRI may address towards diagnosis of ICC when "target appearance" is noted on diffusion weighted imaging or in post-contrast late hepatospecific phase. In the former, DWI images at high b-value will show an hyperintense rim surrounding a central area of hypointensity. In the latter, a central cloud-like hyperintensity will be surrounded by a rim of peripheral hypointensity. In both cases, these typical appearances reflect the histological components of ICC, characterized by a peripheral area of hypercellularity and a central hypocellular area dominated by fibrosis. Histopathology is considered mandatory in order to confirm imaging features which may resemble other benign or malignant (combined) lesions.

\section{Prognostic value of CT and MRI}

Recently, studies have demonstrated that preoperative CT and MRI features - of ICC may predict overall survival and/or disease-free survival after surgical resection or loco-regional therapies.

In ICC, studies suggest that extended areas of arterial hyperenhancement and hyperintense signal on $\mathrm{DWI}^{[46-48]}$ show a favorable prognosis as opposed to tumors characterized by hyper- or isointense signal on the hepatobiliary phase ${ }^{[49]}$ or with extended areas of delayed phase enhancement reflecting a higher fibrous content ${ }^{[50]}$. This is consistent with the histopathological observation that patients with scirrhous type ICC, 
characterized by abundant fibrous stroma, show higher rates of lymph node metastasis and poorer survival outcomes than those with non-scirrhous type ICC ${ }^{[51]}$.

A study by Pandey et al. ${ }^{[52]}$ assessing preoperative characteristics of ICC candidate to trans-arterial chemoembolization demonstrated that overall survival (OS) was higher in patients with lower values of baseline apparent diffusion coefficient, percentage of viable tumor volume $>90 \%$, and viable tumor burden $>6.6 \%$ independently from clinical confounders (age and sex). Data suggest that tumors characterized by lower viable tumor burden, hence a greater degree of fibrosis and necrosis, are associated to a hostile tumor microenvironment, where hypoxia, acidosis, and inadequate perfusion limit the efficacy of intra-arterial drug delivery.

In peCC, a recent study by Yoo et al.$^{[53]}$ on prognostic performance of preoperative MRI demonstrated that imaging evidence of peritumoral fat stranding, common bile duct involvement, and Bismuth type III/IV disease were independent predictors of residual disease after surgery, which was in turn associated to poorer survival.

\section{INTERVENTIONAL RADIOLOGY}

Loco-regional treatments are gaining increasing interest in the field of ICC as technological advances guarantee an effective disease control while maintaining the safety of a minimally invasive approach. Knowledge of CC disease presentation as well as clinical characteristics of the patients are essential to select the most appropriate interventional tool based on a personalized approach. Promising results from combination of loco-regional treatments and systemic therapies of unresectable ICCs are also briefly discussed in this article.

\section{ABLATION}

Ablation is the physical destruction of tumor cells via delivery of thermal energy through a percutaneous needle placement. The low incidence of ICC compared to HCC, makes the availability of literature on the efficacy of ablation scarce. First reported in 2002 by Slakey et al. ${ }^{[54]}$ for the treatment of non-resectable ICC, ablation is now recognized by guidelines as a palliative option for non-resectable ICC measuring $\leq 3 \mathrm{~cm}$ without evidence of extrahepatic disease ${ }^{[55]}$. Kolarich et al. ${ }^{[16]}$, through a population analysis of 4374 patients undergoing non-surgical management of ICC, produced robust evidence in demonstrating that ablation was associated with a statistically significant survival benefit over no local therapy only in stage I disease (i.e., without extrahepatic disease).

Another setting where ablation has shown very interesting results is the management of post-surgical intrahepatic recurrence of ICC. In a recent study by Xu et al. ${ }^{[56]}$ comparing repeated resection $(n=65) v s$. microwave ablation $(n=56)$ of recurrent ICC, with comparable tumor size among the two groups, ablation had a similar efficacy in terms of overall survival and progression free survival with a much lower complication rate.

Ablation can be performed under ultrasound or CT guidance as per local expertise, and several technical options exist for reaching tumor tissue destruction. Among these, radiofrequency ablation is the most extensively studied, with proven technique efficacy in tumors up to $5 \mathrm{~cm}^{[57]}$. Microwave ablation have the advantage of lower susceptibility to heat-sink effects, and with higher temperatures in a short time, it can achieve larger ablation zones ${ }^{[58]}$. However, these theoretical advantages have not effectively translated to an improvement in prognosis ${ }^{[59]}$, leaving the choice among radiofrequency ablation and microwave ablation to local expertise and availability. 
Alternative ablative strategies are cryoablation and irreversible electroporation, both of which may be an option in central liver tumors and tumors adjacent to sensitive structures (e.g., gallbladder, major bile ducts, and bowel loops) due to their safer profile. However, data regarding their efficacy on ICC are extremely scarce $^{[60]}$.

Factors affecting local tumor progression after ablation of ICC are size ${ }^{[61]}$ and superficial location ${ }^{[59]}$. Higher rates of local tumor progression in these settings are the result of under treatment due to technical limitations (larger lesions) and possible damages to neighboring structures (in subcapsular lesions). Factors affecting survival after ablation are albumin-bilirubin grade ${ }^{[62]}$, tumor size, and presence of more than one tumor $^{[59]}$.

One very frequently encountered complication after ablation of ICC is the development of a biliary abscess, with an incidence rate ranging between $7 \%$ and $20 \%{ }^{[63]}$. Risk factors for the occurrence of this complication are cholangiectasis (since thermal injury to the bile duct leads to contamination of ablation zone by enteric bacteria) and presence of bilioenteric anastomosis (due to retrograde enteric bacterial contamination of the biliary tract). For these reasons, pre-procedural care of these patients includes prophylactic antibiotic therapy and palliation of biliary dilation whenever possible.

\section{Transarterial chemoembolization}

Conventional transarterial chemoembolization (cTACE) is the emulsion of chemotherapeutics and an oilbased contrast agent (lipiodol) followed by an embolizing agent into the tumor-feeding artery ${ }^{[64]}$. Doxorubicin, cisplatin, mitomycin-C, and gemcitabine are the most commonly used drug combinations ${ }^{[6,66]}$. In order to be eligible for TACE, good hepatic function (Child-Pugh Class A or B) and performance status ECOG 0-2) are required. As for ablation, in patients with biliary dilation or bilioenteric anastomosis, the increased risk of procedure-related development of biliary abscess justifies a preprocedural prophylactic antibiotic regimen ${ }^{[67]}$. The ideal candidate for TACE is a patient with nonresectable, multifocal disease not tolerating systemic chemotherapy. Another typical indication during MDTs may be the presence of particularly hypervascular disease localization, wherein a good response to intraarterial therapy may be expected.

Survival of ICC after CTACE has been shown to range from 12 to 25.2 months from diagnosis, and from 9.1 to 16.3 months from the procedure ${ }^{[68]}$. The extreme variability in the results is a reflection of the variability in patient-related variables, including tumor burden and previous therapies, as well as technical variables including type of chemotherapeutic drug and number of treatment sessions. CTACE has a safe profile with few adverse events. Post-embolization syndrome (i.e., transient nausea, abdominal pain, fever, and selflimited increase in liver enzymes) ${ }^{[69]}$ is the most common side-effect.

Drug-eluting beads transarterial chemoembolization (DEB-TACE) is a relatively novel advance of TACE, where local release of chemotherapeutic agents is mediated by pre-loaded beads obtaining both a therapeutic and embolizing effect, with lower systemic drug exposure compared $\mathrm{CTACE}^{[70]}$. Also in DEBTACE, efficacy of the procedure is extremely variable, with survival ranging from 8.6 to 30 month ${ }^{[68]}$ with the same limitations described for cTACE.

DEB-TACE has been described with both doxorubicin and irinotecan; a study by Venturini et al. ${ }^{[71]}$ on 10 patients comparing DEB-TACE with doxorubicin and DEB-TACE with irinotecan showed no significant differences between the two groups in terms of safety and efficacy. 
Both in CTACE and DEB-TACE, treatment design may be lobar, segmental, or subsegmental, according to the disease extension and distribution. Underlying liver function must be taken into account when planning the treatment strategy, since lower volume of ischemia may be tolerated in cirrhotic patients; for this reason, treatment schedule may be organized in sequential sessions (at least 2 weeks apart) if a large volume of liver is involved, in order to allow healthy parenchyma to withstand the ischemic injury and preserve function.

\section{Trans-arterial radioembolization}

Trans-arterial radioembolization (TARE) consists in the intra-arterial injection of radioactive microspheres, in order to selectively release a high radiation dose to liver tumor cells while maintaining an acceptable dose to the healthy parenchyma ${ }^{[72]}$. Inclusion criteria for TARE include, but are not limited to, ECOG performance status of 0-2 and adequate liver function with bilirubin $<2.0 \mathrm{mg} / \mathrm{dL}$. Some of the exclusion criteria are the presence of shunts with gastrointestinal arteries not feasible for embolization and an estimated radiation dose to the lungs $>30 \mathrm{~Gy}$. Similar to TACE, the ideal candidates for TARE are patients with unresectable liver-only or liver-dominant tumors ${ }^{[73]}$, particularly if hypervascularization is evident on preoperative imaging.

The procedure is divided in a work-up session and a treatment session, generally a week apart. In the former, an accurate angiographic study, possibly aided by cone-beam-CT technology, is performed to identify and possibly embolize extrahepatic feeders and shunts between hepatic and gastrointestinal circulation. Following a good targeting of the lesion(s), albumin macroaggregates marked with technetium99 (99mTc-MAA) are injected intraarterially and the patient undergoes a single photon emission CT within the same day to map $99 \mathrm{mT}$-MAA distribution and validate treatment feasibility. If the work-up has a positive outcome, the treatment session consists in targeting the same vessels previously injected with 99mTc-MAA and infuse of yttrium-90 (Y-90) microspheres. In case of work-up failure, either a new workup procedure is scheduled or other treatment strategies are considered. TARE may be performed with either glass or resin Y-90 charged microspheres, without significant differences in terms of safety or efficacy ${ }^{[74]}$. In both cases, bead size range is 20-30 microns, which allows radioisotope entrapment within the sinusoids exerting its therapeutic effect by localized radiation rather than by ischemia as seen in TACE. The lower ischemic effect allows the theoretical possibility of treating larger liver volumes than TACE, without altering liver function as much.

Studies show median survivals ranging from 9 to 22 months ${ }^{[67,75]}$; factors associated to worse survival after TARE were having ECOG performance 1 or $2^{[76-78]}$ and infiltrative disease ${ }^{[76,78]}$. However, a considerable heterogeneity of baseline data mainly regarding the inclusion of patients with both peripheral and infiltrative disease, varying tumor burden, and patients who received previous systemic chemotherapy constitute the main limit in drawing conclusions regarding its efficacy.

A meta-regression study by Cucchetti et al. ${ }^{[79]}$ showed that naïve patients had a 2 -year survival of $50.4 \%$ compared to $23.6 \%$ in patients previously treated with systemic CT. Survival was found to be longer in patients with non-infiltrative type ICC; however, these data are not confirmed by the largest single center cohort of patients $(n=85)$ available to date ${ }^{[80]}$. The recent CIRT prospective trial ${ }^{[81]}$ enrolled 120 patients with ICC and showed a median OS of 14.7 months and severe adverse events at thirty days (grade 4 and 5) observed in less than $2.5 \%$ of patients. Of note is that $60.8 \%$ of these patients had received systemic CT.

In addition, TARE seems to have a favorable safety profile: post-radioembolization syndrome, hepatic dysfunction, biliary complications, portal hypertension, radiation pneumonitis, gastrointestinal ulceration, vascular injury, and lymphopenia are the most frequent adverse events ${ }^{[82]}$ even though they rarely occur, 
especially when treatment planning and execution are properly performed.

Another fairly recent interesting application of TARE is in the neoadjuvant setting (i.e., in downstaging an initially unresectable ICC rendering the surgical option feasible). A study by Riby et al. ${ }^{[83]}$ showed that patients undergoing preoperative TARE could undergo resection with a comparable prognosis to patients undergoing upfront surgery; furthermore, TARE showed benefit as downstaging treatment in terms of survival compared with patients treated with chemotherapy.

\section{HEPATIC ARTERY INFUSION CHEMOTHERAPY}

Hepatic arterial infusion chemotherapy (HAIC) (i.e., injecting chemotherapeutic agents into the hepatic artery without embolization) is an option to obtain effective drug delivery without the systemic side effects seen with systemic chemotherapy. HAIC consists of a continuous infusion of floxuridine into the hepatic arterial circulation administered through a surgically implanted pump at a predetermined flow rate ${ }^{[84]}$. Previous studies have demonstrated that HAIC is a promising option for advanced ICC and has shown better tumor control than systemic chemotherapy ${ }^{[8]}$.

Recently, studies involving agents other than floxuridine have been published; a study describing intraarterial epirubicin and cisplatin combined with systemic 5 -fluorouracil demonstrated an objective response rate and median survival time of $36 \%$ and 15.4 months, respectively ${ }^{[86]}$.

A study by Cai et al. ${ }^{\left[{ }^{[7]}\right]}$ compared the outcome of mFOLFOX-HAIC and TACE, demonstrating higher oneyear overall survival rates after the HAIC treatment compared with those after TACE treatment (1-year OS rates: $60.2 \%$ vs. $42.9 \%$, 2 -year OS rates: $38.7 \%$ vs. $29.4 \%, P=0.028$ ).

HAIC was shown to have the highest rate of adverse events and liver-related toxicity among intra-arterial therapies for $\mathrm{ICC}^{[17]}$, therefore it has questionable safety profile. In general, its application is reserved to extended bilobar disease, in case of non-response or poor compliance to systemic chemotherapy.

\section{CHEMOSATURATION - PERCUTANEOUS HEPATIC PERFUSION}

First studied for treatment of hepatic metastases from ocular melanoma ${ }^{[88]}$, chemosaturation - percutaneous hepatic perfusion with intra-arterial melphalan injection represents an emerging technique for disease control in unresectable ICC. In this technique, a transfemoral catheterization of the hepatic artery is used for chemoperfusion of the liver with melphalan. A transfemoral venous double-balloon catheter is inflated to isolate the intrahepatic tract of the inferior vena cava. Venous blood from the liver, extracted through side holes of the double-balloon catheter, is filtered via an extracorporeal hemofiltration circuit, while a transjugular venous access is used for blood return.

A multi-institutional study by Marquardt et al. ${ }^{[89]}$ on a cohort of 26 patients with unresectable ICC demonstrated an overall response rate of $20 \%$ and disease control in $53 \%$ of patients after first treatment session, with a median OS of 26.9 months from initial diagnosis and 7.6 months from first treatment, and median progression free survival of 4.1 months. Patients with liver-only disease exhibited a significantly longer median OS compared to patients with loco-regional lymph node metastases (12.9 months vs. 4.8 months, respectively; $P<0.01$ ). Regarding safety, no grade 3 or 4 adverse events occurred during the procedure; however, hematological toxicity including thrombocytopenia and anemia, requiring transfusions in the post-procedural period, were fairly common, and one patient with a tumor load $>40 \%$ of the liver developed acute multi-organ failure after the treatment. Given its good response coupled with a mild burden of systemic side effects, the application of this treatment strategy is limited to extended liver disease 
not responding to the previously mentioned therapies or systemic therapy.

\section{COMBINED THERAPIES}

Koch et al. ${ }^{\left[{ }^{[0]}\right]}$ showed that combination of TACE and chemotherapy doubled median OS compared to TACE alone (26.3 months). Recently, Mosconi et al. ${ }^{[91]}$ showed that TARE combined with chemotherapy had a median OS of 17.9 months (95\%CI: 14.3-21.4 months) with significantly better median survival in the treatment-naive patients (52 months $v s .16$ months). A phase II clinical trial ${ }^{[92]}$ evaluating TARE plus chemotherapy as first-line for locally advanced ICC in 41 patients, demonstrated a mean OS of 22 months. Interestingly, 9 patients were downstaged to surgery; of these, 8 received resection with Ro margins and 6 were still disease-free at 24 months follow-up. In this study, grade 3-4 hematologic toxicity was observed in $70 \%$ of patients, similarly to the toxicity observed in the ABC-02 trial ${ }^{[93,94]}$, possibly due to gemcitabine/oxaliplatin more than due to addition of TARE. Looking at liver-specific toxicity, among patients with liver cirrhosis $(n=12), 9$ had hepatic failure; of the 9 patients, 5 had undergone bilobar TARE.

HAIC with floxuridine was studied in combination with systemic gemcitabine and oxaliplatin in patients with unresectable ICC ${ }^{[95]}$ in a phase 2 clinical trial on 38 patients, with a mean OS of 25 months; 4 patients were downstaged to surgery. Liver-specific toxicity leading to discontinuation was observed in 4 patients; $47 \%$ received full-dose of floxuridine at 6 months.

\section{CONCLUSION}

The frequency of ICCs, in particular due to its presence in the aging population, is becoming increasingly relevant. Its dismal prognosis, especially in non-resectable disease, makes the role of diagnostic radiology crucial; disease diagnosis at an early stage and the most detailed prognostic information derived from imaging may change the approach and final outcome of patients. As the knowledge of this disease advances, so do treatment strategies, with continuously evolving techniques and indications. In this setting, combination therapies, with the apparent ability to effectively downstage the disease to resectability, seem promising due to a critical improvement compared to both loco-regional treatments alone ${ }^{[17]}$ and to systemic therapy ${ }^{[94]}$. Future perspectives include the introduction of novel systemic agents (i.e., immunotherapeutic regimens and molecular targeted therapy with TACE or TARE). For both the diagnostic and the interventional setting, the multidisciplinary approach, including but not limited to, pathologists, oncologists, and surgeons, is fundamental to achieve a better characterization of the vast CC disease spectrum and identify the best personalized care possible.

\section{DECLARATIONS}

\section{Authors' contributions}

Made substantial contributions to conception and design, data analysis, and interpretation: Della Corte A, Di Gaeta E, Steidler S, De Cobelli F

\section{Availability of data and materials}

Not applicable.

\section{Financial support and sponsorship}

None.

\section{Conflicts of interest}

All authors declare that there are no conflicts of interest. 


\section{Ethical approval and consent to participate}

Not applicable.

\section{Consent for publication}

Not applicable.

\section{Copyright}

(c) The Author(s) 2022.

\section{REFERENCES}

1. Banales JM, Marin JJG, Lamarca A, et al. Cholangiocarcinoma 2020: the next horizon in mechanisms and management. Nat Rev Gastroenterol Hepatol 2020;17:557-88. DOI PubMed PMC

2. Banales JM, Cardinale V, Carpino G, et al. Expert consensus document: Cholangiocarcinoma: current knowledge and future perspectives consensus statement from the European Network for the Study of Cholangiocarcinoma (ENS-CCA). Nat Rev Gastroenterol Hepatol 2016;13:261-80. DOI PubMed

3. Bertuccio P, Malvezzi M, Carioli G, et al. Global trends in mortality from intrahepatic and extrahepatic cholangiocarcinoma. J Hepatol 2019;71:104-14. DOI PubMed

4. Khan SA, Tavolari S, Brandi G. Cholangiocarcinoma: epidemiology and risk factors. Liver Int 2019;39 Suppl 1:19-31. DOI PubMed

5. Jang MH, Lee YJ, Kim H. Intrahepatic cholangiocarcinoma arising in Caroli's disease. Clin Mol Hepatol 2014;20:402-5. DOI PubMed PMC

6. Petrick JL, Yang B, Altekruse SF, et al. Risk factors for intrahepatic and extrahepatic cholangiocarcinoma in the United States: as population-based study in SEER-Medicare. PLoS One 2017;12:e0186643. DOI PubMed PMC

7. Fernández-Ruiz M, Guerra-Vales JM, Colina-Ruizdelgado F. Comorbidity negatively influences prognosis in patients with extrahepatic cholangiocarcinoma. World J Gastroenterol 2009;15:5279-86. DOI PubMed PMC

8. Joo I, Lee JM, Lee SM, Lee JS, Park JY, Han JK. Diagnostic accuracy of liver imaging reporting and data system (LI-RADS) v2014 for intrahepatic mass-forming cholangiocarcinomas in patients with chronic liver disease on gadoxetic acid-enhanced MRI. $J$ Magn Reson Imaging 2016;44:1330-8. DOI PubMed

9. Joo I, Lee JM. Imaging bile duct tumors: pathologic concepts, classification, and early tumor detection. Abdom Imaging 2013;38:133450. DOI PubMed

10. Seo N, Kim SY, Lee SS, et al. Sclerosing cholangitis: clinicopathologic features, imaging spectrum, and systemic approach to differential diagnosis. Korean J Radiol 2016;17:25-38. DOI PubMed PMC

11. Kim JY, Kim MH, Lee TY, et al. Clinical role of 18F-FDG PET-CT in suspected and potentially operable cholangiocarcinoma: a prospective study compared with conventional imaging. Am J Gastroenterol 2008;103:1145-51. DOI PubMed

12. Moon CM, Bang S, Chung JB, et al. Usefulness of 18F-fluorodeoxyglucose positron emission tomography in differential diagnosis and staging of cholangiocarcinomas. J Gastroenterol Hepatol 2008;23:759-65. DOI PubMed

13. Waisberg DR, Pinheiro RS, Nacif LS, et al. Resection for intrahepatic cholangiocellular cancer: new advances. Transl Gastroenterol Hepatol 2018;3:60. DOI PubMed PMC

14. Tamandl D, Herberger B, Gruenberger B, Puhalla H, Klinger M, Gruenberger T. Influence of hepatic resection margin on recurrence and survival in intrahepatic cholangiocarcinoma. Ann Surg Oncol 2008;15:2787-94. DOI PubMed

15. Park JJ, Cheon JH, Kwon JE, et al. Clinical outcomes and factors related to resectability and curability of EMR for early colorectal cancer. Gastrointest Endosc 2011;74:1337-46. DOI PubMed

16. Kolarich AR, Shah JL, George TJ Jr, et al. Non-surgical management of patients with intrahepatic cholangiocarcinoma in the United States, 2004-2015: an NCDB analysis. J Gastrointest Oncol 2018;9:536-45. DOI PubMed PMC

17. Boehm LM, Jayakrishnan TT, Miura JT, et al. Comparative effectiveness of hepatic artery based therapies for unresectable intrahepatic cholangiocarcinoma. J Surg Oncol 2015;111:213-20. DOI PubMed

18. Kuriyama N, Usui M, Gyoten K, et al. Neoadjuvant chemotherapy followed by curative-intent surgery for perihilar cholangiocarcinoma based on its anatomical resectability classification and lymph node status. BMC Cancer 2020;20:405. DOI PubMed PMC

19. Anderson B, Doyle MBM. Surgical considerations of hilar cholangiocarcinoma. Surg Oncol Clin N Am 2019;28:601-17. DOI PubMed

20. Ayuso JR, Pagés M, Darnell A. Imaging bile duct tumors: staging. Abdom Imaging 2013;38:1071-81. DOI PubMed

21. Hennedige TP, Neo WT, Venkatesh SK. Imaging of malignancies of the biliary tract- an update. Cancer Imaging 2014;14:14. DOI PubMed PMC

22. Razumilava N, Gores GJ. Classification, diagnosis, and management of cholangiocarcinoma. Clin Gastroenterol Hepatol 2013;11:1321.e1; quiz e3. DOI PubMed PMC

23. Ruys AT, van Beem BE, Engelbrecht MR, Bipat S, Stoker J, Van Gulik TM. Radiological staging in patients with hilar cholangiocarcinoma: a systematic review and meta-analysis. Br J Radiol 2012;85:1255-62. DOI PubMed PMC

24. Clements O, Eliahoo J, Kim JU, Taylor-Robinson SD, Khan SA. Risk factors for intrahepatic and extrahepatic cholangiocarcinoma: a systematic review and meta-analysis. J Hepatol 2020;72:95-103. DOI PubMed 
25. Uchida M, Ishibashi M, Tomita N, Shinagawa M, Hayabuchi N, Okuda K. Hilar and suprapancreatic cholangiocarcinoma: value of 3D angiography and multiphase fusion images using MDCT. AJR Am J Roentgenol 2005;184:1572-7. DOI PubMed

26. Matos C, Serrao E, Bali MA. Magnetic resonance imaging of biliary tumors. Magn Reson Imaging Clin N Am 2010;18:477-96, x. DOI PubMed

27. Kim HJ, Lee SS, Byun JH, et al. Incremental value of liver MR imaging in patients with potentially curable colorectal hepatic metastasis detected at CT: a prospective comparison of diffusion-weighted imaging, gadoxetic acid-enhanced MR imaging, and a combination of both MR techniques. Radiology 2015;274:712-22. DOI PubMed

28. Lee KH, Lee JM, Park JH, et al. MR imaging in patients with suspected liver metastases: value of liver-specific contrast agent gadoxetic acid. Korean J Radiol 2013;14:894-904. DOI PubMed PMC

29. Shimada K, Isoda H, Hirokawa Y, Arizono S, Shibata T, Togashi K. Comparison of gadolinium-EOB-DTPA-enhanced and diffusionweighted liver MRI for detection of small hepatic metastases. Eur Radiol 2010;20:2690-8. DOI PubMed

30. Olthof SC, Othman A, Clasen S, Schraml C, Nikolaou K, Bongers M. Imaging of cholangiocarcinoma. Visc Med 2016;32:402-10. DOI PubMed PMC

31. Edge S. American Cancer Society. AJCC cancer staging handbook: from the AJCC cancer staging manual. New York: Springer; 2010.

32. Park HS, Lee JM, Choi JY, et al. Preoperative evaluation of bile duct cancer: MRI combined with MR cholangiopancreatography versus MDCT with direct cholangiography. AJR Am J Roentgenol 2008;190:396-405. DOI PubMed

33. Jhaveri KS, Hosseini-Nik H. MRI of cholangiocarcinoma. J Magn Reson Imaging 2015;42:1165-79. DOI PubMed

34. Cui XY, Chen HW. Role of diffusion-weighted magnetic resonance imaging in the diagnosis of extrahepatic cholangiocarcinoma. World J Gastroenterol 2010;16:3196-201. DOI PubMed PMC

35. Razumilava N, Gores GJ, Lindor KD. Cancer surveillance in patients with primary sclerosing cholangitis. Hepatology 2011;54:184252. DOI PubMed PMC

36. Aljiffry M, Abdulelah A, Walsh M, Peltekian K, Alwayn I, Molinari M. Evidence-based approach to cholangiocarcinoma: a systematic review of the current literature. J Am Coll Surg 2009;208:134-47. DOI PubMed

37. Joo I, Lee JM, Yoon JH. Imaging diagnosis of intrahepatic and perihilar cholangiocarcinoma: recent advances and challenges. Radiology 2018;288:7-13. DOI PubMed

38. Choi KS, Lee JM, Joo I, Han JK, Choi BI. Evaluation of perihilar biliary strictures: does DWI provide additional value to conventional MRI? AJR Am J Roentgenol 2015;205:789-96. DOI PubMed

39. Choi EK, Yoo IeR, Kim SH, et al. The clinical value of dual-time point 18F-FDG PET/CT for differentiating extrahepatic cholangiocarcinoma from benign disease. Clin Nucl Med 2013;38:e106-11. DOI PubMed

40. Wang S, Wu H, Wang Q, et al. 18F-FDG PET/CT in differentiating malignant from benign origins of obstructive jaundice. Hepatobiliary Pancreat Dis Int 2015;14:516-22. DOI PubMed

41. Furukawa H, Ikuma H, Asakura-Yokoe K, Uesaka K. Preoperative staging of biliary carcinoma using 18F-fluorodeoxyglucose PET: prospective comparison with PET+CT, MDCT and histopathology. Eur Radiol 2008;18:2841-7. DOI PubMed

42. Anderson CD, Rice MH, Pinson CW, Chapman WC, Chari RS, Delbeke D. Fluorodeoxyglucose PET imaging in the evaluation of gallbladder carcinoma and cholangiocarcinoma. J Gastrointest Surg 2004;8:90-7. DOI PubMed

43. Chong YS, Kim YK, Lee MW, et al. Differentiating mass-forming intrahepatic cholangiocarcinoma from atypical hepatocellular carcinoma using gadoxetic acid-enhanced MRI. Clin Radiol 2012;67:766-73. DOI PubMed

44. Inchingolo R, Maino C, Gatti M, et al. Gadoxetic acid magnetic-enhanced resonance imaging in the diagnosis of cholangiocarcinoma. World J Gastroenterol 2020;26:4261-71. DOI PubMed PMC

45. Oliveira IS, Kilcoyne A, Everett JM, Mino-Kenudson M, Harisinghani MG, Ganesan K. Cholangiocarcinoma: classification, diagnosis, staging, imaging features, and management. Abdom Radiol (NY) 2017;42:1637-49. DOI PubMed

46. Türkoğlu MA, Yamamoto Y, Sugiura T, et al. The favorable prognosis after operative resection of hypervascular intrahepatic cholangiocarcinoma: a clinicopathologic and immunohistochemical study. Surgery 2016;160:683-90. DOI PubMed

47. Ariizumi S, Kotera Y, Takahashi Y, et al. Mass-forming intrahepatic cholangiocarcinoma with marked enhancement on arterial-phase computed tomography reflects favorable surgical outcomes. J Surg Oncol 2011;104:130-9. DOI PubMed

48. Lee J, Kim SH, Kang TW, Song KD, Choi D, Jang KT. Mass-forming intrahepatic cholangiocarcinoma: diffusion-weighted imaging as a preoperative prognostic marker. Radiology 2016;281:119-28. DOI PubMed

49. Koh J, Chung YE, Nahm JH, et al. Intrahepatic mass-forming cholangiocarcinoma: prognostic value of preoperative gadoxetic acidenhanced MRI. Eur Radiol 2016;26:407-16. DOI PubMed

50. Asayama Y, Yoshimitsu K, Irie H, et al. Delayed-phase dynamic CT enhancement as a prognostic factor for mass-forming intrahepatic cholangiocarcinoma. Radiology 2006;238:150-5. DOI PubMed

51. Kajiyama K, Maeda T, Takenaka K, Sugimachi K, Tsuneyoshi M. The significance of stromal desmoplasia in intrahepatic cholangiocarcinoma: a special reference of 'scirrhous-type' and 'nonscirrhous-type' growth. Am J Surg Pathol 1999;23:892-902. DOI PubMed

52. Pandey A, Pandey P, Ghasabeh MA, et al. Baseline volumetric multiparametric MRI: can it be used to predict survival in patients with unresectable intrahepatic cholangiocarcinoma undergoing transcatheter arterial chemoembolization? Radiology 2018;289:843-53. DOI PubMed

53. Yoo J, Kim JH, Bae JS, Kang HJ. Prediction of prognosis and resectability using MR imaging, clinical, and histopathological findings in patients with perihilar cholangiocarcinoma. Abdom Radiol (NY) 2021;46:4159-69. DOI PubMed

54. Slakey DP. Radiofrequency ablation of recurrent cholangiocarcinoma. Am Surg 2002;68:395-7. PubMed

55. Bridgewater J, Galle PR, Khan SA, et al. Guidelines for the diagnosis and management of intrahepatic cholangiocarcinoma. $J$ Hepatol 


\section{4;60:1268-89. DOI PubMed}

56. $\mathrm{Xu} \mathrm{C}, \mathrm{Li} \mathrm{L}, \mathrm{Xu} \mathrm{W}$, et al. Ultrasound-guided percutaneous microwave ablation versus surgical resection for recurrent intrahepatic cholangiocarcinoma: intermediate-term results. Int J Hyperthermia 2019;36:351-8. DOI PubMed

57. Carrafiello G, Laganà D, Cotta E, et al. Radiofrequency ablation of intrahepatic cholangiocarcinoma: preliminary experience. Cardiovasc Intervent Radiol 2010;33:835-9. DOI PubMed

58. Andreano A, Brace CL. A comparison of direct heating during radiofrequency and microwave ablation in ex vivo liver. Cardiovasc Intervent Radiol 2013;36:505-11. DOI PubMed PMC

59. Yousaf A, Kim JU, Eliahoo J, Taylor-Robinson SD, Khan SA. Ablative therapy for unresectable intrahepatic cholangiocarcinoma: a systematic review and meta-analysis. J Clin Exp Hepatol 2019;9:740-8. DOI PubMed PMC

60. Sweeney J, Parikh N, El-Haddad G, Kis B. Ablation of intrahepatic cholangiocarcinoma. Semin Intervent Radiol 2019;36:298-302. DOI PubMed PMC

61. Yu MA, Liang P, Yu XL, et al. Sonography-guided percutaneous microwave ablation of intrahepatic primary cholangiocarcinoma. Eur J Radiol 2011;80:548-52. DOI PubMed

62. Ni JY, An C, Zhang TQ, Huang ZM, Jiang XY, Huang JH. Predictive value of the albumin-bilirubin grade on long-term outcomes of CT-guided percutaneous microwave ablation in intrahepatic cholangiocarcinoma. Int J Hyperthermia 2019;36:328-36. DOI PubMed

63. Yu MA, Liang P, Yu XL, et al. Liver abscess as a complication of microwave ablation for liver metastatic cholangiocarcinoma after bilioenteric anastomosis. Int J Hyperthermia 2011;27:503-9. DOI PubMed

64. Lucatelli P, Burrel M, Guiu B, de Rubeis G, van Delden O, Helmberger T. CIRSE standards of practice on hepatic transarterial chemoembolisation. Cardiovasc Intervent Radiol 2021;44:1851-67. DOI PubMed

65. Kiefer MV, Albert M, McNally M, et al. Chemoembolization of intrahepatic cholangiocarcinoma with cisplatinum, doxorubicin, mitomycin C, ethiodol, and polyvinyl alcohol: a 2-center study. Cancer 2011;117:1498-505. DOI PubMed

66. Shen WF, Zhong W, Liu Q, Sui CJ, Huang YQ, Yang JM. Adjuvant transcatheter arterial chemoembolization for intrahepatic cholangiocarcinoma after curative surgery: retrospective control study. World J Surg 2011;35:2083-91. DOI PubMed

67. Odisio BC, Richter M, Aloia TA, et al. Use of prophylactic antibiotics to prevent abscess formation following hepatic ablation in patients with prior enterobiliary manipulation. J Gastrointest Surg 2016;20:1428-34. DOI PubMed PMC

68. Ierardi AM, Angileri SA, Patella F, et al. The role of interventional radiology in the treatment of intrahepatic cholangiocarcinoma. Med Oncol 2017;34:11. DOI PubMed

69. Cohen MJ, Bloom AI, Barak O, et al. Trans-arterial chemo-embolization is safe and effective for very elderly patients with hepatocellular carcinoma. World J Gastroenterol 2013;19:2521-8. DOI PubMed PMC

70. Aliberti C, Benea G, Tilli M, Fiorentini G. Chemoembolization (TACE) of unresectable intrahepatic cholangiocarcinoma with slowrelease doxorubicin-eluting beads: preliminary results. Cardiovasc Intervent Radiol 2008;31:883-8. DOI PubMed

71. Venturini M, Sallemi C, Agostini G, et al. Chemoembolization with drug eluting beads preloaded with irinotecan (DEBIRI) vs doxorubicin (DEBDOX) as a second line treatment for liver metastases from cholangiocarcinoma: a preliminary study. Br J Radiol 2016;89:20160247. DOI PubMed PMC

72. Kennedy A, Coldwell D, Sangro B, Wasan H, Salem R. Radioembolization for the treatment of liver tumors general principles. Am J Clin Oncol 2012;35:91-9. DOI PubMed

73. Kennedy A, Nag S, Salem R, et al. Recommendations for radioembolization of hepatic malignancies using yttrium-90 microsphere brachytherapy: a consensus panel report from the radioembolization brachytherapy oncology consortium. Int J Radiat Oncol Biol Phys 2007;68:13-23. DOI PubMed

74. Buettner S, Braat AJAT, Margonis GA, et al. Yttrium-90 radioembolization in intrahepatic cholangiocarcinoma: a multicenter retrospective analysis. J Vasc Interv Radiol 2020;31:1035-43.e2. DOI PubMed

75. Al-Adra DP, Gill RS, Axford SJ, Shi X, Kneteman N, Liau SS. Treatment of unresectable intrahepatic cholangiocarcinoma with yttrium-90 radioembolization: a systematic review and pooled analysis. Eur J Surg Oncol 2015;41:120-7. DOI PubMed PMC

76. Ibrahim SM, Mulcahy MF, Lewandowski RJ, et al. Treatment of unresectable cholangiocarcinoma using yttrium-90 microspheres: results from a pilot study. Cancer 2008;113:2119-28. DOI PubMed

77. Hoffmann RT, Paprottka PM, Schön A, et al. Transarterial hepatic yttrium-90 radioembolization in patients with unresectable intrahepatic cholangiocarcinoma: factors associated with prolonged survival. Cardiovasc Intervent Radiol 2012;35:105-16. DOI PubMed

78. Saxena A, Bester L, Chua TC, Chu FC, Morris DL. Yttrium-90 radiotherapy for unresectable intrahepatic cholangiocarcinoma: a preliminary assessment of this novel treatment option. Ann Surg Oncol 2010;17:484-91. DOI PubMed

79. Cucchetti A, Cappelli A, Mosconi C, et al. Improving patient selection for selective internal radiation therapy of intra-hepatic cholangiocarcinoma: a meta-regression study. Liver Int 2017;37:1056-64. DOI PubMed

80. Gangi A, Shah J, Hatfield N, et al. Intrahepatic cholangiocarcinoma treated with transarterial Yttrium-90 glass microsphere radioembolization: results of a single institution retrospective study. J Vasc Interv Radiol 2018;29:1101-8. DOI PubMed PMC

81. Helmberger T, Golfieri R, Pech M, et al; On behalf of the CIRT Steering Committee; On behalf of the CIRT Principal Investigators. Clinical application of trans-arterial radioembolization in hepatic malignancies in Europe: first results from the prospective multicentre observational study CIRSE registry for SIR-spheres therapy (CIRT). Cardiovasc Intervent Radiol 2021;44:21-35. DOI PubMed PMC

82. Riaz A, Lewandowski RJ, Kulik LM, et al. Complications following radioembolization with yttrium-90 microspheres: a comprehensive literature review. J Vasc Interv Radiol 2009;20:1121-30; quiz 1131. DOI PubMed

83. Riby D, Mazzotta AD, Bergeat D, et al. Downstaging with radioembolization or chemotherapy for initially unresectable intrahepatic 
cholangiocarcinoma. Ann Surg Oncol 2020;27:3729-37. DOI PubMed

84. Brown DB, Cardella JF, Sacks D, et al. Quality improvement guidelines for transhepatic arterial chemoembolization, embolization, and chemotherapeutic infusion for hepatic malignancy. J Vasc Interv Radiol 2006;17:225-32. DOI PubMed

85. Kasai K, Kooka Y, Suzuki Y, et al. Efficacy of hepatic arterial infusion chemotherapy using 5-fluorouracil and systemic pegylated interferon $\alpha$-2b for advanced intrahepatic cholangiocarcinoma. Ann Surg Oncol 2014;21:3638-45. DOI PubMed

86. Cantore M, Mambrini A, Fiorentini G, et al. Phase II study of hepatic intraarterial epirubicin and cisplatin, with systemic 5-fluorouracil in patients with unresectable biliary tract tumors. Cancer 2005;103:1402-7. DOI PubMed

87. Cai Z, He C, Zhao C, Lin X. Survival comparisons of hepatic arterial infusion chemotherapy with mFOLFOX and transarterial chemoembolization in patients with unresectable intrahepatic cholangiocarcinoma. Front Oncol 2021;11:611118. DOI PubMed PMC

88. Hughes MS, Zager J, Faries M, et al. Results of a randomized controlled multicenter phase III trial of percutaneous hepatic perfusion compared with best available care for patients with melanoma liver metastases. Ann Surg Oncol 2016;23:1309-19. DOI PubMed $\mathrm{PMC}$

89. Marquardt S, Kirstein MM, Brüning R, et al. Percutaneous hepatic perfusion (chemosaturation) with melphalan in patients with intrahepatic cholangiocarcinoma: European multicentre study on safety, short-term effects and survival. Eur Radiol 2019;29:1882-92. DOI PubMed

90. Koch C, Franzke C, Bechstein WO, et al. Poor prognosis of advanced cholangiocarcinoma: real-world data from a tertiary referral center. Digestion 2020;101:458-65. DOI PubMed

91. Mosconi C, Gramenzi A, Ascanio S, et al. Yttrium-90 radioembolization for unresectable/recurrent intrahepatic cholangiocarcinoma: a survival, efficacy and safety study. Br J Cancer 2016;115:297-302. DOI PubMed PMC

92. Edeline J, Touchefeu Y, Guiu B, et al. Radioembolization plus chemotherapy for first-line treatment of locally advanced intrahepatic cholangiocarcinoma: a phase 2 clinical trial. JAMA Oncol 2020;6:51-9. DOI PubMed PMC

93. Valle J, Wasan H, Palmer DH, et al; ABC-02 Trial Investigators. Cisplatin plus gemcitabine versus gemcitabine for biliary tract cancer. N Engl J Med 2010;362:1273-81. DOI PubMed

94. Lamarca A, Ross P, Wasan HS, et al. Advanced intrahepatic cholangiocarcinoma: post hoc analysis of the ABC-01, -02 , and -03 clinical trials. J Natl Cancer Inst 2020;112:200-10. DOI PubMed

95. Cercek A, Boerner T, Tan BR, et al. Assessment of hepatic arterial infusion of floxuridine in combination with systemic gemcitabine and oxaliplatin in patients with unresectable intrahepatic cholangiocarcinoma: a phase 2 clinical trial. JAMA Oncol 2020;6:60-7. DOI PubMed PMC 